\title{
Growth hormone alleviates oxidative stress and improves the IVF outcomes of poor ovarian responders: a randomized controlled trial
}

Yan Gong ${ }^{1 *}\left(\mathbb{D}\right.$, Kun Zhang ${ }^{2^{*}}$, Dongsheng Xiong ${ }^{1}$, Jiajing Wei ${ }^{1}$, Hao $\operatorname{Tan}^{2}$ and Shengfang Qin $^{3}$

\begin{abstract}
Background: Oxidative stress (OS), defined as an imbalance between excessive reactive oxygen species (ROS) and/ or reactive nitrogen species (RNS) production and antioxidant insufficiency, has been suggested to be involved in the pathogenesis of poor ovarian response (POR). Growth hormone (GH) can reduce OS in some cell types. This study investigated whether GH can improve OS and the in vitro fertilization and embryo transfer (IVF-ET) outcomes of poor ovarian responders.
\end{abstract}

Methods: This study enrolled 105 patients with POR and 58 patients without POR (controls) who were diagnosed according to the Bologna criteria and underwent conventional IVF-ET. Poor ovarian responders were randomly assigned to two groups: the POR-GH group, which received pretreatment with $\mathrm{GH} 4 \mathrm{IU} / \mathrm{d}$ on day 2 of the previous menstrual cycle before IVF until the trigger day, and the POR-C group, which received no pretreatment. OS markers in follicular fluid (FF), ROS levels in granulosa cells (GCs), and the IVF outcomes of the groups were compared.

Results: Endometrial thickness on trigger day, the number of cleaved embryos, the number of higher-quality embryos, and the rates of embryo formation, higher-quality embryo formation, implantation and clinical pregnancy were significantly increased in the POR-GH group compared with the POR-C group $(P<0.05)$. Moreover, compared to those in the non-POR group, FF malondialdehyde (MDA), total oxidant status (TOS), oxidative stress index (OSI) and ROS levels in GCs were significantly higher, whereas superoxide dismutase (SOD) and the total antioxidant capacity (TAC) were significantly lower in the POR-C group $(P<0.05)$. Furthermore, compared with those in the POR-C group, the FF TAC was significantly increased in the POR-GH group, and TOS, OSI and intracellular ROS levels were significantly reduced $(P<0.05)$.

(Continued on next page)

\footnotetext{
*Correspondence: gongyan0619@163.com; zhangkunyyo@163.com

'Reproductive Medicine Centre, Sichuan Provincial Women's and Children's

Hospital, The Affiliated Women's and children's Hospital of Chengdu Medical

College, \#290 Shayan West Second Street, Wuhou District, Chengdu, Sichuan,

People's Republic of China

2Department of Genetics, School of Bioscience and Technology, Chengdu

Medical College, \#783 Xindu Avenue, Xindu District, Chengdu, Sichuan

610500, People's Republic of China

Full list of author information is available at the end of the article
}

(c) The Author(s). 2020 Open Access This article is licensed under a Creative Commons Attribution 4.0 International License, which permits use, sharing, adaptation, distribution and reproduction in any medium or format, as long as you give appropriate credit to the original author(s) and the source, provide a link to the Creative Commons licence, and indicate if changes were made. The images or other third party material in this article are included in the article's Creative Commons licence, unless indicated otherwise in a credit line to the material. If material is not included in the article's Creative Commons licence and your intended use is not permitted by statutory regulation or exceeds the permitted use, you will need to obtain permission directly from the copyright holder. To view a copy of this licence, visit http://creativecommons.org/licenses/by/4.0/ The Creative Commons Public Domain Dedication waiver (http://creativecommons.org/publicdomain/zero/1.0/) applies to the data made available in this article, unless otherwise stated in a credit line to the data. 
(Continued from previous page)

Conclusions: Pretreatment with GH alleviates OS and improves oocyte quality and IVF outcomes of poor ovarian responders.

Trial registration: Chinese Clinical Trial Registry. ChiCTR1900021269. Registered 8 February 2019, http://www.chictr. org.cn/edit.aspx?pid=35837\&htm=4.

Keywords: Poor ovarian response, In vitro fertilization, Growth hormone, Oxidative stress, Reactive oxygen species

\section{Background}

Poor ovarian response (POR) remains one of the major challenges in woman infertility therapy including in vitro fertilization and embryo transfer (IVF-ET). Poor ovarian responders exhibit a higher cycle cancellation rate, produce fewer oocytes and cleaved embryos, and have a lower pregnancy rate and a higher miscarriage rate than individuals without POR [1]. The incidence of POR in IVF-ET ranges from 9 to $24 \%$ and is increasing as women delay childbirth [2]. The IVF outcomes of poor ovarian responders are still low despite the use of different stimulation protocols and multiple treatment courses [3].

Several factors, including advanced female age, ovarian and pelvic surgeries, chemotherapy and radiotherapy, are associated with POR [4]. The physiopathology of POR is complicated and includes follicular loss by atresia/apoptosis, diminished expression of follicle-stimulating hormone (FSH) receptor (FSHR), oocyte chromosomal defects and mitochondrial dysfunction $[5,6]$. Accumulative evidence suggests that oxidative stress (OS) in ovarian ageing is an important pathogenesis of POR [6, 7]. Low to moderate levels of reactive oxygen species (ROS) and/or reactive nitrogen species (RNS) are involved in physiological processes, including defence against infections, cellular signalling systems, and cell growth and differentiation [8]. Excessive ROS/RNS may damage the innate antioxidant defence system and destroy proteins, DNA and lipids. OS is defined as an imbalance between excessive ROS/RNS production and a decrease in antioxidant defence systems in pathologic conditions [9].

Abnormal morphology, dysfunction and a low mitochondrial DNA (mtDNA) copy number have been observed in the oocytes of poor ovarian responders. Mitochondrial dysfunction results in a lower antioxidant production capacity, excessive ROS accumulation and DNA oxidative damage in oocytes, eventually contributing to poor embryo quality and IVF outcomes [10]. Therefore, antioxidant treatment may be beneficial for poor ovarian responders.

A meta-analysis reported that growth hormone (GH) supplementation could significantly improve the clinical pregnancy and live birth rates of poor ovarian responders [3]. The precise mechanism through which GH functions in poor ovarian responders is not fully understood, and most studies focus on its direct or indirect effects via growth hormone receptor (GHR) and insulin-like growth
factor-I (IGF-I) [11]. Studies have revealed that GH augments the effects of gonadotropin on granulosa cells (GCs) and theca cells by binding to GHR and increasing the synthesis of IGF-I to improve follicle development and steroidogenesis [11, 12]. Moreover, Weall et al. [13] reported that GH increased the expression of GHR, improved mitochondrial function and increased the fertilization rate of oocytes among older women. GH can reduce OS in some types of cells [14-16]. However, the ability of GH to improve OS in poor ovarian responders has not been assessed in detail. Obtaining human oocytes for study is very difficult. Remarkably, the oocyte is supported and nourished by intimate cross-talk with its surrounding GCs [17]. The composition of follicular fluid (FF) reflects the metabolic and hormonal processes that occur in the microenvironment of the maturing oocyte [18]. Therefore, FF and GCs can be used as surrogate bioassays to study the biological processes of oocytes.

Based on the above evidence, this prospective, randomized, controlled study investigated the effects of GH on markers of OS in FF and GCs and the IVF outcomes of poor ovarian responders.

\section{Materials and methods}

\section{Ethics statements}

This prospective, randomized, open-label study was registered in the Chinese Clinical Trial Registry Centre (Registration No. ChiCTR1900021269) and approved by the Medical Ethics Committee of Sichuan Provincial Women's and Children's Hospital. Signed informed consent forms were obtained from all participants. All procedures in this study complied with the ethical standards of the relevant national and institutional committees on human experimentation and with the Helsinki Declaration 1975 (2013 revision).

The sample size calculation was based on the assumption that the clinical pregnancy rate would increase threefold after GH pretreatment. The clinical pregnancy rate of the poor ovarian responders in our centre was approximately $13 \%$, and 48 patients were required for each group, with an $\alpha$ of 0.05 and a $\beta$ error of 0.1 (power $=90 \%$ ).

\section{Study subjects}

Patients with POR (aged 33-43 years) diagnosed according to the Bologna criteria [1] who underwent IVF were 
enrolled from the Reproductive Medicine Centre of Sichuan Provincial Women's and Children's Hospital (between Feb. 2019 and Dec. 2019). The poor ovarian responders were randomized 1:1 to either $\mathrm{GH}$ pretreatment (the POR-GH group) or no GH pretreatment (the POR-C group) (using computer-generated random numbers). The exclusion criteria were as follows: (1) hydrosalpinx, congenital uterine malformations and/or endometrial disease such as tuberculosis or hyperplasia; (2) a basal follicle-stimulating hormone (bFSH) level $\geq 15$ IU/L; (3) systemic lupus erythematosus, sicca syndrome, or polycystic ovarian syndrome; (4) an uncontrolled endocrinopathy such as diabetes, hyperthyroidism, hypothyroidism, or hyperprolactinemia; (5) IVF-ET treatment within three months; (6) an intracytoplasmic sperm injection (ICSI) cycle due to male infertility; or (7) supplementation with any antioxidants such as vitamin E, vitamin C, CoQ10, beta-carotene or selenium. Women with tubal factor infertility (aged 20-35 years) with a normal ovarian reserve and regular menstrual cycles who underwent IVF-ET were recruited as non-POR controls during the same period. The exclusion criteria for the non-POR group were the same as those for the POR group.

Data pertaining to the medical history of each participant were collected, including the regularity of menstrual cycles, the duration of infertility, and treatment history. Abnormal menstrual cycles included oligomenorrhoea, polymenorrhoea, irregular menstrual cycles, and amenorrhoea. Body mass index (BMI) was calculated as body weight divided by the square of body height $\left(\mathrm{kg} / \mathrm{m}^{2}\right)$. The antral follicle count (AFC) was determined by transvaginal ultrasound on days $2-3$ of menstruation or progestin-induced bleeding withdrawal.

\section{Controlled ovarian stimulation (COS) and IVF}

All patients underwent controlled ovarian stimulation (COS) with the GnRH antagonist protocol. Recombinant follicle-stimulating hormone ( $\mathrm{rFSH}$ ) (Gonal-F; MerckSerono KGaA., Darmstadt, Germany) was injected on day 2 of the menstrual cycle, and rFSH doses were adjusted according to follicle growth and serum hormone levels. In the POR-GH group, $4 \mathrm{IU} / \mathrm{d}$ of recombinant human growth hormone (Changchun GeneScience Pharmaceuticals Co., Ltd., Changchun, Jilin, China) was injected subcutaneously on day 2 of the previous menstrual cycle before IVF until the trigger day (36-48 days). When a leading follicle reached $12 \mathrm{~mm}$ and/or serum $E_{2}$ levels reached $300 \mathrm{pg} / \mathrm{mL}$, Ganirelix (Merck Sharp \& Dohme co., Ltd., Hoddesdon, United Kingdom) was administered. When at least one follicle was greater than $18 \mathrm{~mm}$, recombinant human chorionic gonadotropin (hCG) (Ovitrelle ${ }^{\oplus}$; Merck-Serono KGaA) was administered as the trigger. When no follicles with diameters $\geq 14 \mathrm{~mm}$ were noted after 10 days of gonadotrophin injection or when the peak $E_{2}$ level was below $300 \mathrm{pg} /$ $\mathrm{mL}$, the cycle was cancelled. Ultrasound-guided transvaginal oocyte retrieval was performed $36 \mathrm{~h}$ later, and follicle flushing was not performed. Upon oocyte retrieval, FF was collected only from follicles with a diameter $\geq 16$ $\mathrm{mm}$ measured on the retrieval day. FF samples were immediately centrifuged at $700 \mathrm{~g}$ for $5 \mathrm{~min}$ at room temperature, and the supernatant was stored at $-80^{\circ} \mathrm{C}$. The precipitates were suspended in $3 \mathrm{~mL}$ of PBS, gently layered in $3 \mathrm{~mL} 50 \%$ lymphocyte separation medium (Beijing Solarbio Science and Technology Corporation, Beijing, Solarbio Science and Technology Co., Ltd., China) and then centrifuged at $700 \mathrm{~g}$ for $10 \mathrm{~min}$ to remove red blood cells and debris. GCs layered at the interface of the gradient were washed twice with $5 \mathrm{~mL}$ of PBS (Nanjing KeyGen Biotech. Co., Ltd., Nanjing, Jiangsu, ChinaKeyGEN Bio TECH Co., Ltd., Jiangsu, China) and immediately examined for ROS using fluorescence microscopy and spectrophotometry. FF or GCs from each patient were collected separately and considered to be one sample. According to the criteria established by the Istanbul Consensus Workshop on Embryo Assessment, the cultured embryos on day 3 were assessed based on the number of blastomeres and the degree of fragmentation, and higher-quality embryos were categorized as grade A/B [19]. One or two fresh embryos with the best morphological grade were selected for transfer. The remaining embryos were all cultured to the blastocyst stage to be frozen. Luteal phase support with intramuscular injection of progesterone 60 $\mathrm{mg} / \mathrm{d}$ was initiated on the oocyte retrieval day. Serum hCG was measured 12 days after ET, and hCG positivity was considered when hCG $>5 \mathrm{IU} / \mathrm{mL}$. Clinical pregnancy was defined as demonstration of a gestational sac with an embryo showing cardiac activity. Early miscarriage was defined as loss of pregnancy before gestational week 12. Ovarian hyperstimulation syndrome (OHSS) was diagnosed according to Navot D et al. [20].

The maturation rate was calculated as the number of MII oocytes divided by the number of oocytes retrieved. The fertilization rate was calculated as the number of two pronuclear zygotes (2PN) divided by the number of MII oocytes. The embryo formation rate was calculated as the number of day 3 embryos divided by the number of $2 \mathrm{PN}$. The higher-quality embryo formation rate was calculated as the number of higher-quality embryos divided by the number of 2PN. The implantation rate was calculated as the observed number of gestational sacs divided by the number of transferred embryos. The clinical pregnancy rate was expressed as the number of clinical pregnancy cycles divided by the number of embryo transfer cycles. The miscarriage rate was expressed as the number of spontaneous pregnancy loss cycles 
before 12 weeks of gestation divided by the number of clinical pregnancy cycles.

We suggest $\mathrm{GH} 4 \mathrm{IU} / \mathrm{d}$ pretreatment on day 2 of the previous menstrual cycle before IVF until the trigger day because a low physiological dose and longer treatment (from the antral follicle stage) might be more beneficial to follicular growth and development [2].

\section{Measurement of endocrine and metabolic parameters}

Plasma glucose was measured using the hexokinase method (Beijing Strong Biotechnologies, Inc., Beijing, China). Oestradiol $\left(\mathrm{E}_{2}\right)$, progesterone $(\mathrm{P})$, total testosterone (TT), luteinizing hormone (LH), FSH, and insulin levels were measured using the electrochemiluminescence immunoassay platform (Roche Diagnostics GmbH, Mannheim, Germany). The serum level of anti-Mullerian hormone $(\mathrm{AMH})$ was measured using an enzyme-linked immunosorbent assay kit (Guangzhou Kangrun Biotech, Co., Ltd., Guangdong, China). The homeostasis model assessment (HOMA-IR) index was calculated as fasting glucose $(\mathrm{mmol} / \mathrm{L}) \times$ fasting insulin $(\mathrm{mU} / \mathrm{L}) / 22.5$ [21]. The intra- and inter-assay coefficients of the above variation were less than 5 and 10\%, respectively.

\section{OS marker in FF assay procedures}

FF malondialdehyde (MDA) concentrations ( $\mu \mathrm{mol} / \mathrm{L})$ were measured using micro-MDA detection kits (NanJing Jiancheng Bioengineering Institute Co. Ltd., Nanjing, Jiangsu, China), and ultraviolet spectrophotometry (Shanghai Meipuda Instrument Co., Ltd., Shanghai, China) was performed at $532 \mathrm{~nm}$. Superoxide dismutase (SOD) was determined using SOD kits (NanJing Jiancheng Bioengineering Institute Co. Ltd.) and spectrophotometry at $450 \mathrm{~nm}$. The total antioxidant capacity (TAC) (mmol Trolox Equiv./L) and TOS ( $\mu$ mol $\mathrm{H}_{2} \mathrm{O}_{2}$ Equiv./L) were measured by the semiautomatic microplate colorimetric method using hydrogen peroxide $\left(\mathrm{H}_{2} \mathrm{O}_{2}\right)$ and Trolox, respectively, for the standard [22, 23]. The oxidative stress index (OSI) was expressed as the ratio of TOS to the TAC. The measurements were performed in duplicate for each OS parameter. Serum samples from healthy volunteers were pooled for quality control. The intra- and inter-assay coefficients of the above variations were less than 5 and $10 \%$, respectively.

\section{Detection of intracellular ROS levels}

With a dichlorodihydrofluorescein diacetate (DCFH-DA) fluorescent probe, ROS levels in GCs were detected by ROS assay kit (Beyotime Biotechnology Co., Ltd., Shanghai, China). Briefly, GCs were resuspended in PBS and incubated with $10 \mu \mathrm{mol} / \mathrm{L}$ DCFH-DA in the dark for $25 \mathrm{~min}$ $\left(37^{\circ} \mathrm{C}\right)$ and then incubated together with $10 \mu \mathrm{g} / \mathrm{mL} 4^{\prime}, 6-$ diamidino-2-phenylindole (DAPI) (NeoFroxx, Frankfurt, Germany) for $5 \mathrm{~min}$. After the cells were washed three times with PBS, GC suspensions were added to glass slides, and fluorescence was examined by fluorescence microscopy (Olympus Corporation, Tokyo, Japan). The examination wavelength was $488 \mathrm{~nm}$, and the emission wavelength was $525 \mathrm{~nm}$.

Similar to the above protocol but without DAPI, another set of GCs was used to measure the intracellular ROS level by NanoDrop UV-Vis spectrophotometry (Thermo Scientific, Massachusetts, USA). The fluorescence intensities are shown as the intensity in the POR group relative to that in the control group (non-POR group).

\section{Statistical analysis}

All data were statistically analysed using SPSS 17.0 software (SPSS Inc., Chicago IL, USA). Continuous variables were expressed as the mean \pm standard deviation (SD). The Kolmogorov-Smirnov test was used to assess the normality of the data distribution. Continuous variables with normal distributions were compared using the Student-Newman-Keuls test, and Bonferroni's test was used as the post hoc test. Categorical data were compared using chi-squared tests. A two-tailed $P \leq 0.05$ was considered statistically significant.

\section{Results}

\section{Clinical, baseline endocrine and metabolic characteristics} of the study population

The flow diagram of participant selection is shown in Fig. 1. Among poor ovarian responders, 38 had risk factors for POR (advanced age, abnormal menstrual cycles and ovarian surgery history), 44 had a previous POR three months prior ( $\leq 3$ oocytes with a conventional stimulation protocol), and 71 had an abnormal ovarian reserve test (ORT) $(\mathrm{AFC}<5-7$ and/or $\mathrm{AMH}<0.5-1.1$ $\mathrm{ng} / \mathrm{mL}$ ). Compared to those in the non-POR group, age, basal $\mathrm{FSH}$, the $\mathrm{FSH} / \mathrm{LH}$ ratio and $\mathrm{bE}_{2}$ were significantly higher, whereas AFC and AMH were significantly lower in the POR-GH group and POR-C group $(P<0.05)$. The clinical, endocrine and metabolic characteristics did not significantly differ between the POR-GH and POR-C groups $(P>0.05)$ (Table 1$)$.

\section{COS and IVF outcomes were improved in the POR-GH group}

In the POR-GH group, 3 cycles were excluded because no oocytes were retrieved or because fertilization failed. In the POR-C group, 8 cycles were excluded because of the absence of follicular growth, failed oocyte retrieval or failed fertilization. Compared to those in the nonPOR group, the rFSH duration, $\mathrm{E}_{2}$ levels on trigger day, the number of retrieved oocytes, the number of MII oocytes, the number of fertilized oocytes, the number of cleaved embryos and the number of higher-quality embryos were significantly lower in the POR-GH group 
CONSORT Flow Diagram

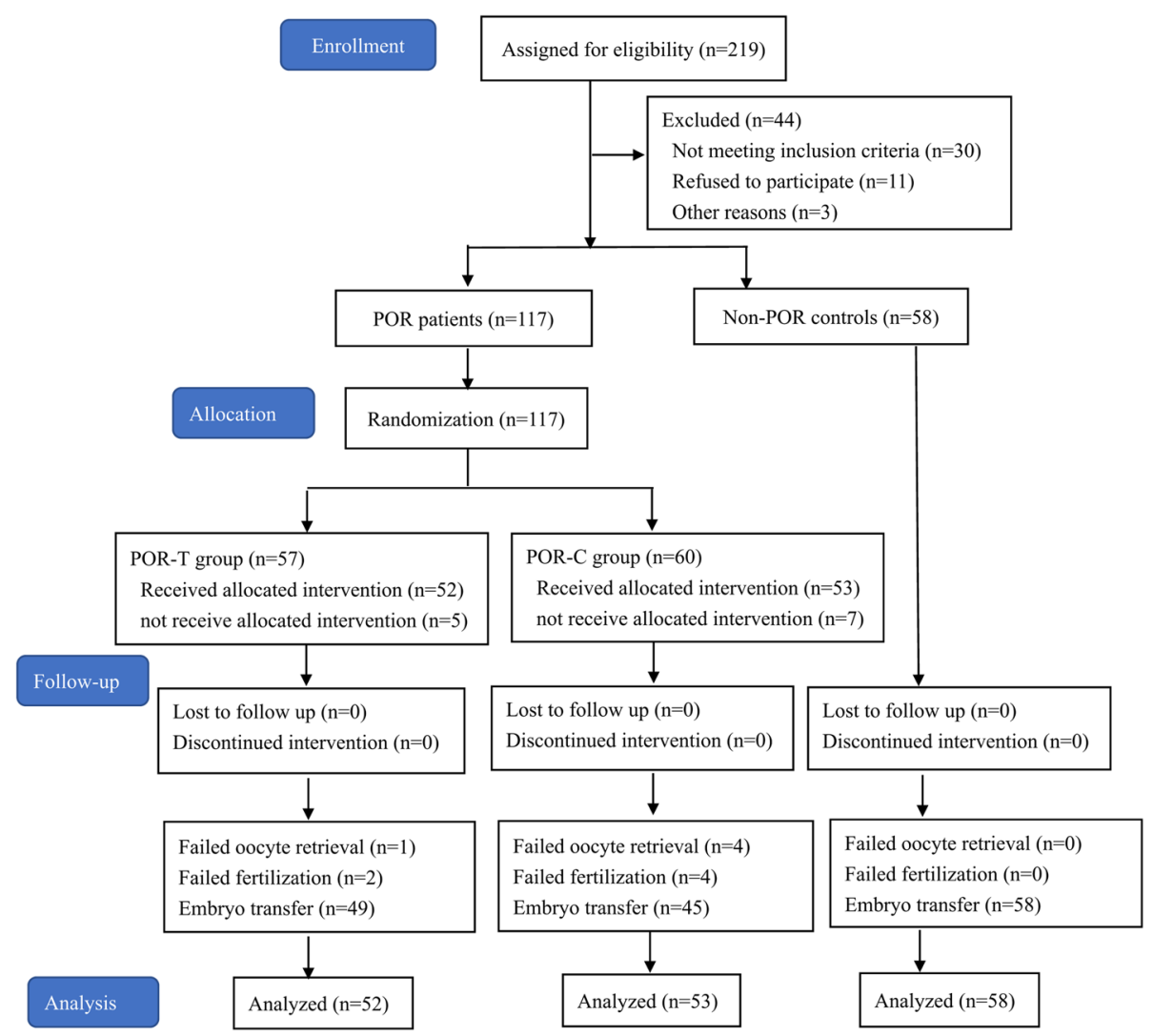

Fig. 1 Flow diagram of this randomized controlled trial. Progression from recruitment to completion

and POR-C group, but the rFSH doses were significantly higher $(P<0.05)$. The endometrial thickness on trigger day, implantation rate and clinical pregnancy rate were significantly reduced, whereas the excluded cycle rate was significantly increased in the POR-C group compared with the non-POR group $(P<0.05)$. Endometrial thickness $(9.65 \pm 1.84$ vs. $8.61 \pm 1.23 \mathrm{~mm})$, the number of cleaved embryos ( $2.31 \pm 1.81$ vs. $1.73 \pm 1.03)$, the number of high-quality embryos $(1.26 \pm 0.65$ vs. $0.72 \pm 0.56)$, the embryo formation rate $(95.76 \%$ vs. $83.87 \%)$, the higherquality embryo formation rate $(52.54 \%$ vs. $35.48 \%)$, the implantation rate $(28.21 \%$ vs. $9.72 \%)$ and the clinical pregnancy rate $(38.77 \%$ vs. $13.33 \%)$ were significantly increased in the POR-GH group compared with the POR$C$ group $(P<0.05)$. No side effect or moderate or severe OHSS was reported in the study population (Table 2).

The levels of OS markers in the FF of the POR-GH group were decreased

Compared to those in the non-POR group, MDA, TOS and OSI levels in FF were significantly higher in the POR-GH group and POR-C group, but the levels of SOD and TAC were significantly lower $(P<0.05)$. The
TAC $(0.59 \pm 0.13$ vs. $0.42 \pm 0.16)$ was significantly increased, whereas TOS $(8.06 \pm 1.19$ vs. $10.14 \pm 4.86)$ and the OSI $(16.49 \pm 8.19$ vs. $23.87 \pm 10.13)$ were significantly decreased in the POR-GH group compared with the POR-C group $(P<0.05)$ (Table 2$)$.

\section{ROS accumulation in GCs of the POR-GH group was inhibited}

The green fluorescence intensity of ROS in GCs was significantly higher in the POR-GH group and POR-C group than in the non-POR group. Additionally, the fluorescence intensity of ROS $(2.36 \pm 0.32$ vs. $1.00 \pm 0.23)$ was significantly higher in the POR-C group than in the non-POR group $(P<0.05)$. Furthermore, ROS intensity $(1.83 \pm 0.38$ vs. $2.36 \pm 0.32$ ) was significantly reduced in the POR-GH group compared with the POR-C group $(P<0.05)$. The fluorescence intensity of ROS is expressed as the fold change relative to the control (Fig. 2).

\section{Discussion}

This study found the existence of an OS state in the FF and GCs of poor ovarian responders undergoing IVF. $\mathrm{GH}$ pretreatment lowered the TOS and OSI in FF and 
Table 1 The clinical, basal endocrine and metabolic characteristics of the study population

\begin{tabular}{|c|c|c|c|}
\hline & POR-GH $(\boldsymbol{n}=52)$ & POR-C $(\boldsymbol{n}=53)$ & Non-POR $(\boldsymbol{n}=58)$ \\
\hline Age (years) ${ }^{a b}$ & $38.41 \pm 2.91$ & $38.20 \pm 2.79$ & $29.56 \pm 3.12$ \\
\hline Infertility duration (years) & $4.57 \pm 3.14$ & $4.39 \pm 3.22$ & $3.47 \pm 2.22$ \\
\hline Risk factors for POR cycle (n) ${ }^{\text {ab }}$ & 20 & 18 & 0 \\
\hline Abnormal ORT cycle (n) ${ }^{a b}$ & 39 & 32 & 0 \\
\hline Previous POR cycle (n) ${ }^{a b}$ & 21 & 23 & 0 \\
\hline BMI $\left(\mathrm{kg} / \mathrm{m}^{2}\right)$ & $22.09 \pm 1.73$ & $22.62 \pm 2.73$ & $22.19 \pm 2.67$ \\
\hline $\mathrm{FSH}(\mathrm{mlU} / \mathrm{mL})^{\mathrm{ab}}$ & $11.49 \pm 2.94$ & $10.97 \pm 2.75$ & $5.89 \pm 1.76$ \\
\hline LH (mlU/mL) & $4.59 \pm 1.83$ & $4.36 \pm 1.57$ & $4.29 \pm 1.45$ \\
\hline $\mathrm{FSH} / \mathrm{LH}$ ratio ab & $2.74 \pm 1.57$ & $2.53 \pm 1.68$ & $1.38 \pm 0.75$ \\
\hline$E_{2}(p g / m L){ }^{a b}$ & $75.98 \pm 34.05$ & $72.34 \pm 26.87$ & $40.62 \pm 11.19$ \\
\hline$P(n g / m L)$ & $0.64 \pm 0.39$ & $0.54 \pm 0.35$ & $0.55 \pm 0.20$ \\
\hline$\Pi T(\mathrm{ng} / \mathrm{mL})$ & $0.37 \pm 0.19$ & $0.41 \pm 0.20$ & $0.43 \pm 0.15$ \\
\hline $\mathrm{AMH}(\mathrm{ng} / \mathrm{mL}){ }^{\mathrm{ab}}$ & $0.88 \pm 0.67$ & $0.81 \pm 0.41$ & $3.50 \pm 1.91$ \\
\hline PRL $(\mu \mid \mathrm{U} / \mathrm{mL})$ & $162.59 \pm 50.19$ & $171.25 \pm 52.32$ & $163.51 \pm 45.79$ \\
\hline HOMA-IR & $1.68 \pm 1.25$ & $1.71 \pm 1.34$ & $1.67 \pm 1.33$ \\
\hline AFC ${ }^{a b}$ & $4.12 \pm 1.71$ & $4.06 \pm 2.11$ & $15.30 \pm 4.56$ \\
\hline
\end{tabular}

Note: Data are presented as the mean \pm SD or number. $B M I$ body mass index, $F S H$ follicle-stimulating hormone, $L H$ luteinizing hormone, $E_{2}$ oestradiol, $P$ progesterone, $\Pi T$ total testosterone, $A M H$ anti-Mullerian hormone, $P R L$ prolactin, HOMA-IR homeostatic model assessment of insulin resistance, $A F C$ antral follicle count

a $P<0.05$ the POR-GH group versus the non-POR group

${ }^{b} P<0.05$ the POR-C group versus the non-POR group

intracellular ROS levels but increased the TAC in FF, suggesting that GH may alleviate OS in the FF and GCs of poor ovarian responders. We also found that GH improved the endometrial thickness on trigger day, increased the number of cleaved embryos, and improved embryo quality, the implantation rate and the clinical pregnancy rate. Alleviating OS may be one of the mechanisms by which GH improves oocyte quality and the IVF outcomes of poor ovarian responders.

We found that the levels of MDA, TOS and OSI in FF were higher, but that the levels of SOD and TAC were lower in poor ovarian responders. Numerous studies have demonstrated the OS state in these patients [6, 24, 25]. The TAC represents the ability to eliminate free radicals. Oyawoye et al. reported [26] that the optimum value of the TAC in $\mathrm{FF}$ was $0.68 \mathrm{mmol} / \mathrm{L}$ for fertilization and subsequent early zygote development, and that the TAC was decreased in women over 37 years old. Similarly, we found that the TAC was $0.71 \pm 0.11 \mathrm{mmol}$ Trolox Equiv./L in patients without POR and decreased to $0.42 \pm 0.16 \mathrm{mmol}$ Trolox Equiv./L in poor ovarian responders. MDA is one of the end-products of lipid peroxidation. TOS represents antioxidant compounds, and the OSI represents the relative level of OS. Interestingly, we found that GH significantly increased the TAC and decreased the values for TOS and the OSI in FF, with more cleaved embryos and higher-quality embryos. FF plays a critical role in oocyte development; therefore, alleviating OS in FF may improve oocyte quality and IVF outcomes $[25,27]$. The above results suggested that GH could improve oocyte quality possibly by suppressing the OS level in FF.

To further assess the OS state in the ovary, we examined the level of ROS in GCs. We found that intracellular ROS levels were significantly higher in poor ovarian responders. GCs are steroidogenic cells surrounding the oocyte. Their metabolic activity is driven by mitochondria and is assumed to provide nutrients and maturation-enabling factors to oocytes [28]. Therefore, GCs play an important role in oocyte development, ovulation, fertilization, and ROS accumulation [29]. Low doses of ROS are important signals for oocyte maturation and ovulation. Under normal physiological conditions, GCs protect oocytes from OS via antioxidant systems, such as $\mathrm{SOD}$ and $\mathrm{E}_{2}$ [30]. In poor ovarian responders, the antioxidant defence system is suppressed, and more abnormal mitochondrial formation and dysfunction are found in GCs [30]. Furthermore, COS induces ROS production in GCs due to active metabolic and proliferation processes [31]. In general, the above factors trigger GC apoptosis and impair oocyte quality in poor ovarian responders [32]. Here, we found that GH effectively decreased ROS levels in GCs. Our result is consistent with the antioxidant effects of $\mathrm{GH}$ in other types of cells, including vascular endothelial cells, myocardial cells and skeletal muscle cells [14-16], suggesting a possible mechanism by which GH could improve oocyte quality. 
Table 2 Controlled ovarian stimulation, IVF outcomes and OS markers in FF

\begin{tabular}{|c|c|c|c|}
\hline & POR-GH $(\boldsymbol{n}=52)$ & POR-C $(\boldsymbol{n}=53)$ & Non-POR $(\boldsymbol{n}=58)$ \\
\hline rFSH doses (IU) $)^{a, b}$ & $2491.46 \pm 996.47$ & $2499.87 \pm 1345.16$ & $1875.09 \pm 467.39$ \\
\hline rFSH duration $(d)$ a,b & $8.93 \pm 2.57$ & $8.97 \pm 4.11$ & $10.65 \pm 1.65$ \\
\hline$E_{2}$ on trigger day $(p g / m L) ~ a, b$ & $985.13 \pm 348.44$ & $887.85 \pm 372.21$ & $1956.10 \pm 558.48$ \\
\hline Endometrial thickness $(\mathrm{mm}){ }^{\mathrm{b}, \mathrm{c}}$ & $9.65 \pm 1.84$ & $8.61 \pm 1.23$ & $10.33 \pm 1.93$ \\
\hline Oocytes retrieved $\mathrm{a,b}$ & $3.71 \pm 2.50$ & $3.24 \pm 2.56$ & $12.51 \pm 6.81$ \\
\hline MIl oocytes $a, b$ & $3.18 \pm 1.78$ & $2.87 \pm 1.89$ & $9.95 \pm 4.28$ \\
\hline Fertilized oocytes (2PN) ${ }^{a, b}$ & $2.36 \pm 1.86$ & $2.02 \pm 1.21$ & $7.04 \pm 4.29$ \\
\hline Cleaved embryos $a, b, c$ & $2.31 \pm 1.81$ & $1.73 \pm 1.03$ & $5.38 \pm 3.62$ \\
\hline Higher-quality embryos ${ }^{a, b, c}$ & $1.26 \pm 0.65$ & $0.72 \pm 0.56$ & $3.34 \pm 2.78$ \\
\hline Oocyte maturation rate (\%) & $82.38 \%(159 / 193)$ & $83.02 \%(132 / 159)$ & $79.48 \%(577 / 726)$ \\
\hline Oocyte fertilization rate & $74.21 \%(118 / 159)$ & $70.45 \%(93 / 132)$ & $70.71 \%(408 / 577)$ \\
\hline Embryo formation rate ${ }^{a, c}$ & $95.76 \%(113 / 118)$ & $83.87 \%(78 / 93)$ & $76.47 \%(312 / 408)$ \\
\hline Higher-quality embryo formation rate $b, c$ & $52.54 \%(62 / 118)$ & $35.48 \%(33 / 93)$ & $47.55 \%(194 / 408)$ \\
\hline No. ET & $1.58 \pm 0.51$ & $1.59 \pm 0.48$ & $1.43 \pm 0.50$ \\
\hline Excluded cycle rate (\%) ${ }^{b}$ & $5.77 \%(3 / 52)$ & $15.09 \%(8 / 53)$ & $0 / 58$ \\
\hline Implantation rate (\%) ${ }^{\mathrm{b}, \mathrm{c}}$ & $28.21 \%(22 / 78)$ & $9.72 \%(7 / 72)$ & $43.37 \%(36 / 83)$ \\
\hline Clinical pregnancy rate (\%) ${ }^{b, c}$ & $38.77 \%(19 / 49)$ & $13.33 \%(6 / 45)$ & $53.44 \%(31 / 58)$ \\
\hline Miscarriage rate (\%) & $21.05 \%(4 / 19)$ & $33.33 \%(2 / 6)$ & $6.45 \%(2 / 31)$ \\
\hline Multiple pregnancy rate (\%) & $15.78 \%(3 / 19)$ & $16.67 \%(1 / 6)$ & $16.13 \%(5 / 31)$ \\
\hline \multicolumn{4}{|l|}{ In FF } \\
\hline $\mathrm{MDA}(\mathrm{nmol} / \mathrm{mL})^{\mathrm{a}, \mathrm{b}}$ & $2.27 \pm 0.69$ & $3.02 \pm 1.10$ & $1.33 \pm 0.67$ \\
\hline SOD (U/mgprot) $)^{a, b}$ & $12.99 \pm 2.32$ & $11.21 \pm 1.71$ & $14.76 \pm 1.93$ \\
\hline TAC (mmol Trolox Equiv./L) ${ }^{a, b, c}$ & $0.59 \pm 0.13$ & $0.42 \pm 0.16$ & $0.71 \pm 0.11$ \\
\hline $\operatorname{TOS}\left(\mu \mathrm{mol} \mathrm{H}_{2} \mathrm{O}_{2} \text { Equiv./L) }\right)^{\mathrm{a}, \mathrm{b}, \mathrm{c}}$ & $8.06 \pm 1.19$ & $10.14 \pm 4.86$ & $5.67 \pm 1.09$ \\
\hline OS| $\left.\right|^{a, b, c}$ & $16.49 \pm 8.19$ & $23.87 \pm 10.13$ & $8.16 \pm 1.86$ \\
\hline
\end{tabular}

Note: Data are presented as the mean \pm SD or percentage (number). $2 P N$ Number of two pronuclear zygotes, ET embryos transferred, $F F$ follicular fluid, MDA malondialdehyde, SOD superoxide dismutase TAC total antioxidant capacity, TOS total oxidant status, OSI oxidative stress index. A chi-squared test was performed to compare the rates of cycle cancellation, implantation, clinical pregnancy, miscarriage and multiple pregnancy

a $P<0.05$ the POR-GH group versus the non-POR group

${ }^{b} P<0.05$ the POR-C group versus the non-POR group

c $P<0.05$ the POR-GH group versus the POR-C group

We found that GH could increase the number of cleaved embryos and embryo quality; however, GH may not affect the number of retrieved oocytes, MII oocytes or fertilized oocytes. GH may improve the quality but not quantity of oocytes by alleviating OS and enhancing mitochondrial function [13]. However, other studies have reported that more overall and fertilized oocytes were achieved by GH treatment, but that no difference was observed in embryo quality [33]. These discrepant results may be due to heterogeneity of the GH protocol, different COS protocols, or inconsistent POR definitions between studies.

In this study, we found that GH improved endometrial thickness in poor ovarian responders. The uterus is a site in which both $\mathrm{GH}$ production and $\mathrm{GH}$ actions are observed [11]. The glandular cells of the human endometrium and decidual tissue express GHR [34]. A series of studies have confirmed that GH increases endometrial blood circulation, expression of receptivity related genes and cytokines, as result to improve endometrial thickness and receptivity [35, 36]. Endometrial receptivity is an important factor for embryo implantation [36]. Therefore, the effects on endometrial tissue may be another mechanism by which GH affects the IVF outcomes of poor ovarian responders.

In addition to the above benefits, we found that $\mathrm{GH}$ could improve the implantation and clinical pregnancy rates of poor ovarian responders. However, another study reported inconsistent results, namely, that GH could not improve the clinical pregnancy rate [37]. We also found that the miscarriage rate was lower in poor ovarian responders pretreated with $\mathrm{GH}$, but the difference was not significant. However, Tesarik J et al. [33] reported that GH significantly decreased the miscarriage 
a
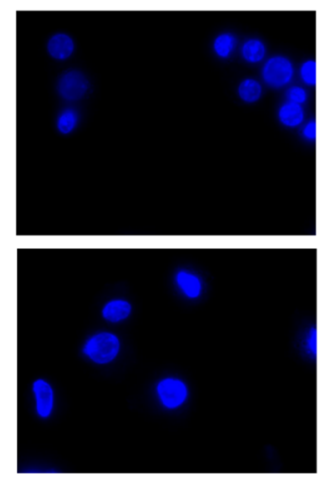

a2
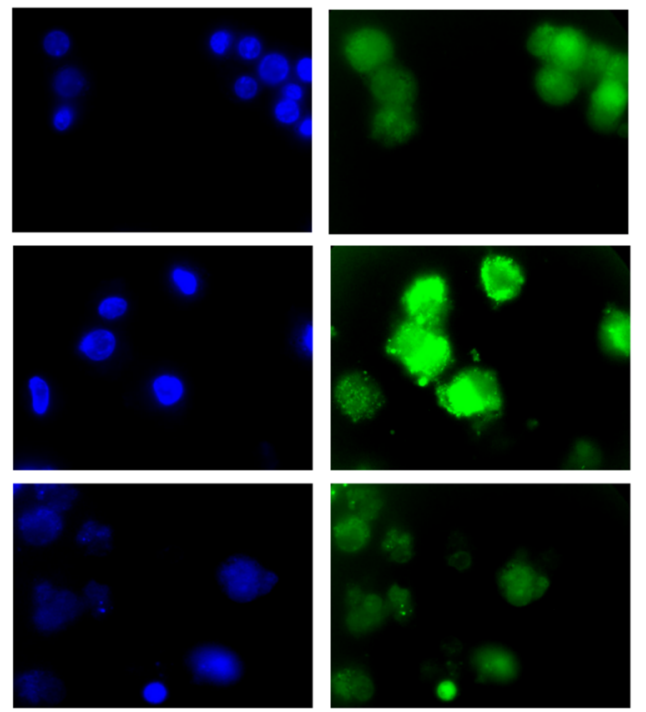

\section{b}

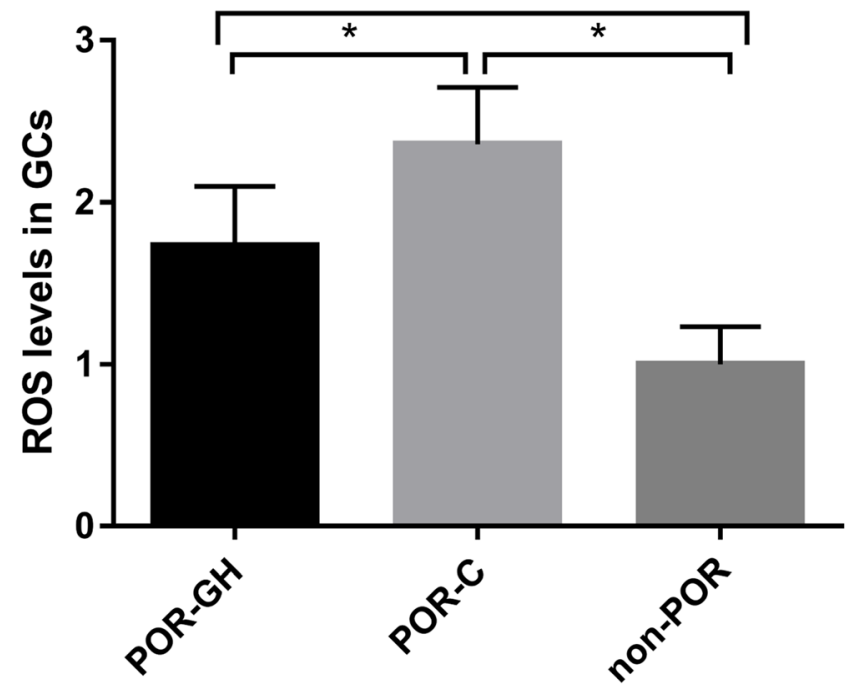

b merge
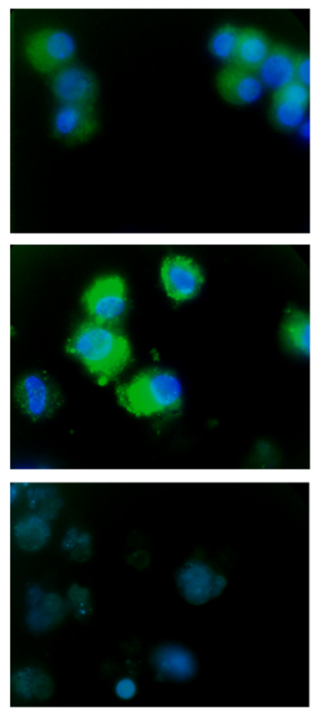

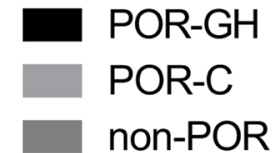

Fig. 2 Fluorescence of ROS in GCs of the three groups. a The green fluorescence of ROS in GCs was observed under a fluorescence microscope. Cell nuclei were stained with DAPI. a1: POR-GH group, a2: POR-C group, a3: non-POR group. $\mathbf{b}$ The fluorescence intensity of ROS is expressed as the fold change relative to the control. Measured by spectrophotometry, the fluorescence intensity of ROS (2.36 $\pm 0.32 \mathrm{vs}$. $1.00 \pm 0.23)$ was significantly higher in the POR-C group than in the non-POR group $(P<0.05)$. GH significantly lowered ROS intensity in the POR-GH group $(1.83 \pm$ 0.38 vs. $2.36 \pm 0.32)(P<0.05)$

rate. These conflicting results can be explained by the discrepancies between the studies.

There were limitations in this study. The sample size was small, and the average age of the patients was 38 years; thus, the results should be analysed and interpreted with caution. Moreover, due to limitations of the study period, we could not evaluate the effect of GH on the live birth rate, which is a more favourable indicator of IVF outcomes. However, whether GH can improve the live birth rate is still controversial $[2,38]$.

\section{Conclusion}

In conclusion, this study revealed high levels of OS in poor ovarian responders undergoing IVF. GH pretreatment alleviated OS, improved oocyte quality and IVF outcomes in these patients. 


\section{Acknowledgements}

We thank our colleagues at the Reproductive Medicine Centre for their assistance in sample collection. We thank Xiang-Yu Li for the data analysis. We very much appreciate all the patients who participated in this study.

\section{Authors' contributions}

Yan Gong designed the study, measured the oxidative stress markers, and wrote the manuscript. Kun Zhang contributed to data analyzing and article revising. Hao Tan and Sheng-fang Qin contributed to the measuring of intracellular reactive oxygen species. Jia-jing Wei and Dong-sheng Xiong participated in sample collection. All authors read and approved the final manuscript.

\section{Funding}

This study was funded by the Technology Innovation Project of Science and Technology Bureau of Chengdu (2018-YF05-00247-SN and 2018-YF05-00672SN), the Scientific Research Project of Sichuan Medical Association (S17060), the Science and Technology Innovation Fund of Sichuan Provincial Hospital for Women and Children (20180205) and the Innovation and Entrepreneurship Training Program for College Students of China (No. 508-2043140).

\section{Availability of data and materials}

The datasets used and/or analysed during the current study are available from the corresponding author upon reasonable request.

\section{Ethics approval and consent to participate}

Approval was obtained from the Medical Ethics Committee of Sichuan Provincial Hospital for Woman and Children. The procedures used in this study adhere to the tenets of the Declaration of Helsinki.

\section{Consent for publication}

Not applicable.

\section{Competing interests}

The authors declare that they have no conflict of interest.

\section{Author details}

'Reproductive Medicine Centre, Sichuan Provincial Women's and Children's Hospital, The Affiliated Women's and children's Hospital of Chengdu Medical College, \#290 Shayan West Second Street, Wuhou District, Chengdu, Sichuan, People's Republic of China. ${ }^{2}$ Department of Genetics, School of Bioscience and Technology, Chengdu Medical College, \#783 Xindu Avenue, Xindu District, Chengdu, Sichuan 610500, People's Republic of China. ${ }^{3}$ Department of Medical Genetics and Prenatal Diagnosis, Sichuan Provincial Women's and Children's Hospital, The Affiliated Women's and children's Hospital of Chengdu Medical College, Chengdu, Sichuan, People's Republic of China.

Received: 12 March 2020 Accepted: 27 August 2020

Published online: 05 September 2020

\section{References}

1. Ferraretti AP, La Marca a, Fauser BC, Tarlatzis B, Nargund G, Gianaroli L. Definition EwgoPOR. ESHRE consensus on the definition of 'poor response' to ovarian stimulation for in vitro fertilization: the Bologna criteria. Hum Reprod. 2011:26:1616-24

2. Kolibianakis EM, Venetis CA, Diedrich K, Tarlatzis BC, Griesinger G. Addition of growth hormone to gonadotrophins in ovarian stimulation of poor responders treated by in-vitro fertilization: a systematic review and metaanalysis. Hum Reprod Update. 2009:15:613-22.

3. Jeve YB, Bhandari HM. Effective treatment protocol for poor ovarian response: a systematic review and meta-analysis. J Hum Reprod Sci. 2016;9: 70-81.

4. Kyrou D, Kolibianakis EM, Venetis CA, Papanikolaou EG, Bontis J, Tarlatzis BC How to improve the probability of pregnancy in poor responders undergoing in vitro fertilization: a systematic review and meta-analysis. Fertil Steril. 2009:91:749-66.

5. Eichenlaub-Ritter U. Oocyte ageing and its cellular basis. Int J Dev Biol. 2012 56:841-52.

6. Tatone C, Amicarelli F, Carbone MC, Monteleone P, Caserta D, Marci R, et al. Cellular and molecular aspects of ovarian follicle ageing. Hum Reprod Update. 2008;14:131-42.
7. Luddi A, Capaldo A, Focarelli R, Gori M, Morgante G, Piomboni P, et al Antioxidants reduce oxidative stress in follicular fluid of aged women undergoing IVF. Reprod Biol Endocrinol. 2016;14:57.

8. Murri M, Luque-Ramirez M, Insenser M, Ojeda-Ojeda M, Escobar-Morreale HF. Circulating markers of oxidative stress and polycystic ovary syndrome (PCOS): a systematic review and meta-analysis. Hum Reprod Update. 2013; 19:268-88.

9. Aruoma Ol, Grootveld M, Bahorun T. Free radicals in biology and medicine: from inflammation to biotechnology. Biofactors. 2006;27:1-3.

10. Kregel $\mathrm{KC}$, Zhang $\mathrm{HJ}$. An integrated view of oxidative stress in aging: basic mechanisms, functional effects, and pathological considerations. Am J Physiol Regul Integr Comp Physiol. 2007;292:R18-36

11. Hull KL, Harvey S. Growth hormone and reproduction: a review of endocrine and autocrine/paracrine interactions. Int J Endocrinol 2014, 2014: 234014

12. Alviggi C, Humaidan P, Howles CM, Tredway D, Hillier SG. Biological versus chronological ovarian age: implications for assisted reproductive technology. Reprod Biol Endocrinol. 2009;7:101.

13. Weall BM, Al-Samerria S, Conceicao J, Yovich JL, Almahbobi G. A direct action for $\mathrm{GH}$ in improvement of oocyte quality in poor-responder patients. Reproduction. 2015:149:147-54.

14. Suzuki K, Yanagi K, Shimizu M, Wakamatsu S, Niitani T, Hosonuma S, et al. Effect of growth hormone replacement therapy on plasma diacron-reactive oxygen metabolites and endothelial function in Japanese patients: the GREAT clinical study. Endocr J. 2018:65:101-11.

15. Brioche T, Kireev RA, Cuesta S, Gratas-Delamarche A, Tresguerres JA, GomezCabrera MC, et al. Growth hormone replacement therapy prevents sarcopenia by a dual mechanism: improvement of protein balance and of antioxidant defenses. J Gerontol A Biol Sci Med Sci. 2014;69:1186-98.

16. Kato $Y$, Iwase M, Ichihara S, Kanazawa H, Hashimoto K, Noda A, et al. Beneficial effects of growth hormone-releasing peptide on myocardial oxidative stress and left ventricular dysfunction in dilated cardiomyopathic hamsters. Circ J. 2010;74:163-70.

17. Li R, Albertini DF. The road to maturation: somatic cell interaction and selforganization of the mammalian oocyte. Nat Rev Mol Cell Biol. 2013;14:141-52.

18. Barroso G, Barrionuevo M, Rao P, Graham L, Danforth D, Huey S, et al. Vascular endothelial growth factor, nitric oxide, and leptin follicular fluid levels correlate negatively with embryo quality in IVF patients. Fertil Steril. 1999;72:1024-6.

19. Alpha Scientists in Reproductive M, Embryology ESIGo. The Istanbul consensus workshop on embryo assessment: proceedings of an expert meeting. Hum Reprod. 2011;26:1270-83.

20. Navot D, Bergh PA, Laufer N. Reprint of: ovarian hyperstimulation syndrome in novel reproductive technologies: prevention and treatment. Fertil Steril. 2019:112:e209-21.

21. Yang S, Wang Q, Huang W, Song Y, Feng G, Zhou L, et al. Are serum chemerin levels different between obese and non-obese polycystic ovary syndrome women? Gynecol Endocrinol. 2016;32:38-41.

22. Zhang R, Liu H, Bai H, Zhang Y, Liu Q, Guan L, et al. Oxidative stress status in Chinese women with different clinical phenotypes of polycystic ovary syndrome. Clin Endocrinol. 2017:86:88-96.

23. Zhou M, Liu XH, Liu QQ, Chen M, Bai H, Guan LB, et al. Lactonase activity, status, and genetic variations of Paraoxonase 1 in women with gestational diabetes mellitus. J Diabetes Res 2020, 2020:3483427.

24. Fujimoto VY, Bloom MS, Huddleston HG, Shelley WB, Ocque AJ, Browne RW. Correlations of follicular fluid oxidative stress biomarkers and enzyme activities with embryo morphology parameters during in vitro fertilization. Fertil Steril. 2011:96:1357-61.

25. Siristatidis C, Vogiatzi P, Varounis C, Askoxylaki M, Chrelias C, Papantoniou N. The effect of reactive oxygen species on embryo quality in IVF. Vivo. 2016; 30:149-53.

26. Oyawoye OA, Abdel-Gadir A, Garner A, Leonard AJ, Perrett C, Hardiman P. The interaction between follicular fluid total antioxidant capacity, infertility and early reproductive outcomes during in vitro fertilization. Redox Rep. 2009;14:205-13

27. Scalici E, Traver S, Molinari N, Mullet T, Monforte M, Vintejoux E, et al. Cellfree DNA in human follicular fluid as a biomarker of embryo quality. Hum Reprod. 2014:29:2661-9.

28. Uyar A, Torrealday S, Seli E. Cumulus and granulosa cell markers of oocyte and embryo quality. Fertil Steril. 2013:99:979-97.

29. Adashi EY. Endocrinology of the ovary. Hum Reprod. 1994;9:815-27. 
30. Carbone MC, Tatone C, Delle Monache S, Marci R, Caserta D, Colonna R, et al. Antioxidant enzymatic defences in human follicular fluid: characterization and age-dependent changes. Mol Hum Reprod. 2003;9: 639-43.

31. Aurrekoetxea I, Ruiz-Sanz Jl, del Agua AR, Navarro R, Hernandez ML, Matorras $\mathrm{R}$, et al. Serum oxidizability and antioxidant status in patients undergoing in vitro fertilization. Fertil Steril. 2010;94:1279-86.

32. Agarwal A, Aponte-Mellado A, Premkumar BJ, Shaman A, Gupta S. The effects of oxidative stress on female reproduction: a review. Reprod Biol Endocrinol. 2012;10:49.

33. Tesarik J, Hazout A, Mendoza C. Improvement of delivery and live birth rates after ICSI in women aged $>40$ years by ovarian co-stimulation with growth hormone. Hum Reprod. 2005;20:2536-41.

34. Sharara FI, Nieman LK. Growth hormone receptor messenger ribonucleic acid expression in leiomyoma and surrounding myometrium. Am J Obstet Gynecol. 1995;173:814-9.

35. Xue-Mei W, Hong J, Wen-Xiang Z, Yang L. The effects of growth hormone on clinical outcomes after frozen-thawed embryo transfer. Int I Gynaecol Obstet. 2016;133:347-50.

36. Cui N, Li AM, Luo ZY, Zhao ZM, Xu YM, Zhang J, et al. Effects of growth hormone on pregnancy rates of patients with thin endometrium. J Endocrinol Investig. 2019;42:27-35.

37. Eftekhar M, Aflatoonian A, Mohammadian F, Eftekhar T. Adjuvant growth hormone therapy in antagonist protocol in poor responders undergoing assisted reproductive technology. Arch Gynecol Obstet. 2013;287:1017-21.

38. Norman RJ, Alvino H, Hull LM, Mol BW, Hart RJ, Kelly TL, et al. Human growth hormone for poor responders: a randomized placebo-controlled trial provides no evidence for improved live birth rate. Reprod BioMed Online. 2019;38:908-15.

\section{Publisher's Note}

Springer Nature remains neutral with regard to jurisdictional claims in published maps and institutional affiliations.

Ready to submit your research? Choose BMC and benefit from:

- fast, convenient online submission

- thorough peer review by experienced researchers in your field

- rapid publication on acceptance

- support for research data, including large and complex data types

- gold Open Access which fosters wider collaboration and increased citations

- maximum visibility for your research: over $100 \mathrm{M}$ website views per year

At $\mathrm{BMC}$, research is always in progress.

Learn more biomedcentral.com/submissions 